\title{
Prostatectomía paliativa en cáncer de próstata localmente avanzado y metastásico con uropatía obstructiva
}

\section{Palliative Prostatectomy of Locally Advanced and Metastatic Prostate Cancer with Obstructive Uropathy}

\author{
Julián Chavarriaga Soto ${ }^{1}$ David Álvarez Villarraga ${ }^{2}$ \\ Juliana Álvarez Jaramillo ${ }^{4}$ Andrés Felipe Quiñones ${ }^{5}$ \\ ${ }^{1}$ Residente de Urología, Pontificia Universidad Javeriana, Bogotá, Colombia \\ 2 Urólogo, Pontificia Universidad Javeriana; Docente Urología, \\ Facultad de Ciencias de la Salud, Programa de Medicina, Universidad \\ Libre, seccional Cali, Departamento de Urología Clínica DESA, Clínica \\ Nueva Rafael Uribe Uribe, Cali, Colombia \\ 3 Urólogo, Pontificia Universidad Javeriana, Hospital Universitario \\ San Ignacio; Profesor Asistente, Facultad de Medicina, Universidad \\ Javeriana, Bogotá, Colombia \\ 4 Universidad de la Sabana. Clínica DESA, Cali, Colombia \\ 5 Pontificia Universidad Javeriana, Bogotá, Colombia \\ ${ }^{6}$ Universidad del Rosario, Bogotá, Colombia
}

José Miguel Silva Herrera ${ }^{3}$ Nayib Fakih Garcia ${ }^{1}$ María Antonia Ocampo ${ }^{6}$ Juan David Iregui ${ }^{2}$

Address for correspondence Julián Chavarriaga Soto, MD, Transversal 2\#67-22, Bogotá Colombia (e-mail: chavarriagaj@javeriana.edu.co).

\section{Resumen}

\section{Palabras Clave}

- Cáncer de próstata

- Prostatectomía

- Uropatía obstructiva

- Calidad de vida

- Síntomas urinarios obstructivos bajos
Introducción Los pacientes con cáncer de próstata localmente avanzado frecuentemente consultan por síntomas urinarios obstructivos bajos secundarios a la obstrucción del tracto de salida de la vejiga por el efecto obstructivo del adenocarcinoma prostático. El objetivo de este estudio es evaluar la prostatectomía transuretral paliativa en esos pacientes y comparar las características preoperatorias, calidad de vida e IPSS antes de la cirugía y en el postoperatorio.

Métodos y Materiales Estudio retrospectivo multicentrico que mediante el cuestionario WHOQOL-Bref evalúa calidad de vida y compara el IPSS pre y postquirúrgico en una cohorte de 26 pacientes llevados a Resección transuretral de la próstata paliativa (pRTUP) por Cáncer de próstata localmente avanzado.

Resultados La media de la edad poblacional fue de 73 años, todos fueron llevados a PRTUP entre el 2011 y el 2017, solo un paciente fue dado de alta el mismo día de la cirugía, la media de hospitalización de 2,1 días. Cinco pacientes (26\%), tenía antecedente de pRTUP y requirieron una nueva intervención. La media del IPSS inicial fue $23.2 \pm 5.4$. El Qmax antes de la intervención quirúrgica tuvo una media de $8.3 \pm 1$, más del $50 \%$ de los pacientes eran de alto riesgo dado por el Gleason. Con respecto a la QoL encontramos que en los dominios de salud física y psicológica fue moderada, mientras que en los otros dominios fue adecuada, el IPSS postquirúrgico mejoro considerablemente con una media de $16.5 \pm 3.4$. received

September 1, 2017

accepted

November 7, 2017

published online

May 11, 2018
DOI https://doi.org/

$10.1055 / \mathrm{s}-0038-1651512$.

ISSN 0120-789X.

eISSN 2027-0119.
Copyright $\odot$ 2018, Sociedad Colombiana License terms de Urología. Publicado por Thieme Revinter Publicações Ltda., Rio de Janeiro, Brazil. Todos los derechos reservados.

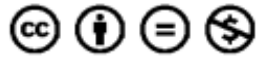




\section{Abstract}

\section{Keywords}

- prostate cancer

- prostatectomy

- obstructive uropathy

- quality of life

- lower urinary tract symptoms
Conclusiones La pRTUP es una buena opción de tratamiento para la desobstrucción al tracto de salida de la vejiga secundaria al adenocarcinoma de próstata localmente avanzado, con mejoría considerable de los síntomas urinarios obstructivos bajos, el IPSS y con buena calidad de vida después de la intervención. Sin evidencia de progresión oncológica de la enfermedad secundaria a la intervención quirúrgica desobstructiva.

Introduction Patients with locally advanced prostate cancer usually complaint of lower urinary tract symptoms secondary to bladder outlet obstruction as a consequence of obstructive uropathy due to prostate adenocarcinoma growth. The aim of this study is to evaluate QoL, IPSS, perioperative characteristics and safety of the palliative transurethral prostatectomy.

Methods Retrospective study that asses 20 men with histopathological confirmed diagnosis of locally advanced prostate cancer and LUTS who underwent palliative prostatectomy.

Results The average way of our simple was 73 years, alla of our patients underwent PTURP between 2011 and 2017, only one patient was discharged the same day of the procedure, the average internation time was 2.1 days, 5 patients (26\%) had already underwent pTURP and needed a second time for palliation of the LUTS. Average initial IPSS was $23.2 \pm 5.4$, the Qmax before the surgery was $8.3 \pm 1$ and the majority of patients were classified as high risk according to D́amico classification system due to the gleason score. Regards the QoL we found that in the physical and psychologic health domains the average score was low while in the other domains was considerably better. Postoperative IPSS was $16.5 \pm 3.4$.

Conclusion PTURP can be performed in patients with locally advanced prostate cancer and LUTS safely and effective with improvement in quality of life, micturition parameters and LUTS, without evidence that the surgical intervention increases oncologic progression of the disease.

\section{Introducción}

La queja más frecuente en pacientes con cáncer de próstata localmente avanzado, son los síntomas urinarios bajos (SUOB), secundarios a la obstrucción al tracto de salida de la vejiga, lo que puede llevar a la retención urinaria, residuo postmiccional elevado, cistolitiasis e inclusive hidronefrosis bilateral secundaria a la uropatía obstructiva asociada al adenocarcinoma prostático. Una de las limitaciones más grandes de esos pacientes con estadio avanzado del cáncer de próstata, es que no tiene opciones curativas de tratamiento; por lo que las conductas a seguir son netamente paliativas con la finalidad de mejorar la calidad de vida que no inciden en la mortalidad especifica asociada al cáncer, ni tampoco en la sobrevida libre de enfermedad. Bajo esas circunstancias, las opciones de tratamiento son: terapia de privación androgénica y derivación de la vía urinaria, procedimientos mínimamente invasivos (Laser, Stent), resección transuretral prostática paliativa (pRTUP), prostatectomía con láser de diodo, y HPS $120 \mathrm{~W}$ Green laser. Esas conductas han sido controversiales a pesar de que se vienen utilizando desde varias décadas atrás, la razón es que se ha propuesto un efecto oncológico negativo durante la cirugía desobstructiva por diseminación intravascular de las células cancerígenas en el seno venoso cuando se utilizan altas presiones de irrigación. ${ }^{1,2}$

A pesar de que la pRTUP se utiliza como parte del tratamiento paliativo en cáncer de próstata localmente avanzado con uropatía obstructiva, hasta la fecha no hay suficientes estudios en las publicaciones internacionales que evalúen específicamente dicha prostatectomía paliativa y no contamos con ningún estudio de esa índole en Colombia.

Nuestro objetivo es revisar el estado de la cuestion de la pRTUP y la prostatectomía endoscópica con láser en el paciente con cáncer de próstata localmente avanzado y metastasico y presentar una serie de casos con el respectivo análisis de calidad de vida y control oncológico. Queremos con eso mostrar la evidencia local que permita determinar las características preoperatorias de ese grupo de pacientes, la morbilidad operatoria y los desenlaces postoperatorios.

\section{Métodos y Materiales}

Este estudio nace ante las inquietudes que despierta el paciente con cáncer de próstata localmente avanzado, no candidato para opciones curativas, con síntomas urinarios 
obstructivos bajos (SUOB) y ante la falta de recomendaciones precisas en las guías de práctica clínica. El abordaje más frecuentemente usado es la pRTUP. Pretende evaluar las características perioperatorias, morbilidad, complicaciones y desenlaces de la resección transuretral prostática paliativa en pacientes con cáncer de próstata localmente avanzado, al caracterizar la población de estudio (edad, raza, etc.), y las condiciones perioperatorias relativas al IPSS y QoL.

La muestra consta de 26 hombres con cáncer de próstata de las características ya descritas, llevados a pRTUP en el Hospital Universitario San Ignacio, la Clínica de Diagnósticos Especializados S.A- DESA y la Clínica Nueva Rafael Uribe Uribe; algunos con terapia de privación hormonal o vigilancia pasiva/activa. Por lo tanto, es un estudio multicéntrico retrospectivo que analiza una cohorte intervenida entre enero del 2011 y marzo de 2017; Se evaluó a los pacientes con el IPSS antes de la intervención quirúrgica y el IPSS posterior a la intervención que se realizó entre uno y dos meses después de haber realizado el procedimiento quirúrgico. Se contactó de manera telefónica a los pacientes en marzo de 2017 para realizar el cuestionario WHOQOL-BREF.

Los criterios de inclusión fueron hombres entre 45 y 85 años, con diagnóstico de cáncer de próstata localmente avanzado (T3NXMX), no candidatos a manejo con intención curativa, pacientes con los anteriores criterios y un IPSS moderado a severo sin respuesta a terapia con manejo farmacológico; se excluyeron a los pacientes que cumplían los siguientes criterios: pacientes con IPSS leve que no habían recibido terapia farmacológica, cáncer de próstata avanzado con compromiso de la vejiga, diagnostico confirmado de vejiga neurogénica hiporreflexica o arreflexica, condición neurológica que impidiera firmar consentimiento informado.

El estudio fue previamente aprobado por los comités de ética e investigación de los diferentes centros asistenciales. Los procedimientos fueron llevados a cabo por dos cirujanos en la ciudad de Cali y dos cirujanos en la ciudad de Bogotá, con la técnica quirúrgica estándar de Nesbit, sin ninguna modificación de la misma. Para analizar los datos se realiza un análisis univariado utilizando medidas de variabilidad como la desviación estándar y medidas de tendencia central como la media aritmética.

\section{Resultados}

La muestra completa fue un total de 26 pacientes que cumplían los criterios del estudio entre los tres centros de atención en salud. De los 26 pacientes siete habían fallecido o no fue posible contactarlos.

El promedio de edad fue de 73 años, todos con diagnóstico de adenocarcinoma de próstata al momento de la RTUP. Sólo un paciente fue dado de alta el mismo día de la cirugía, todos los otros pacientes continuaron hospitalizados con una media de hospitalización de 2.1 días. Cinco pacientes (26\%) tenian antecedente de pRTUP y requirieron una nueva intervención para la paliación de los síntomas obstructivos del tracto urinario bajo. La cirugía se llevó a cabo con la técnica descrita por Nesbit, la media del tiempo quirúrgico fue
86,8 minutos, la media de sangrado fue $246 \mathrm{cc}$, pero no en todos los pacientes se contaba con la medición volumétrica de la próstata sin embargo, en todos estaban descritos los campos de resección endoscópica, la media fue de 1,82 campos y teniendo en cuenta el reporte de patología con respecto al volumen resecado la media fue de $19.6 \mathrm{cc}$. Solo en un caso se presentó una complicación que requirió Reintervención quirúrgica por sangrado y shock hipovolémico, transfusión de 3 unidades de glóbulos rojos empaquetados, lográndose así controlar el sangrado y el procedimiento fue exitoso.

La media del IPSS inicial fue $23,2 \pm 5,4$, así 17 pacientes (89\%) tenían un IPSS severo antes de la cirugía y solo 2 (11\%) un IPSS moderado, pero con importante compromiso de la calidad de vida. Con respecto al octavo ítem del IPSS que evalúa el efecto en la calidad de vida secundaria a síntomas urinarios, la media fue $4,1 \pm 1,4$ sobre 5 .

El Qmax antes de la intervención quirúrgica tuvo una media de $8,3 \pm 1$ sin embargo, muchas de las uroflujometrías se calificaron como equivocadas por obtener un volumen insuficiente. En cuanto a la estatificación inicial, 6 pacientes eran $\mathrm{T} 1 \mathrm{c}(31 \%), 2$ pacientes $\mathrm{T} 2 \mathrm{~b}, 6$ (31\%) mayor a T3, y en el resto no era conocido el TNM inicial y nueve pacientes tenían enfermedad metastásica (47\%).

En cuanto al puntaje de Gleason, 5 pacientes tenían un Gleason $3+3$ score 6,3 un puntaje de 7, dos, $3+4$ y uno, $4+3$, siete pacientes tenían gleason score 9 y los otros un score de 8 , como podemos observar la mayoría de los casos eran de alto riesgo por el Gleason.

La aproximación inicial ocurrió así: cinco recibieron radioterapia con intención curativa con posterior recaída bioquímica y 14 tratados sin intención curativa (-Tabla $\mathbf{1})$.

La calidad de vida en el postoperatorio se evaluó con un cuestionario diseñado por la Organización mundial de la Salud (OMS) el WHOQOL- BREF un cuestionario de 26 preguntas que se puntúa de 0 a 100 y que se divide en cinco dominios: salud física, salud psicológica, relaciones relaciones sociales, entorno y calidad de vida. Encontramos que la media en cada dominio fue: 58,3 para el dominio de calidad de vida, 59,4 en salud física, 57,5 en salud psicológica, 67,1 en relaciones sociales y 62,2 en entorno. Llama la atención que los puntajes más bajos se obtuvieron en salud física y salud psicológica lo que puede corresponder a la condición de enfermo del paciente y saber que tiene que vivir con el diagnóstico de cáncer de próstata localmente avanzado o metastásico. La calidad de vida tampoco estuvo bien punteada sin embargo, se debería comparar esa cohorte con una de pacientes que no han sido sometidos a pRTUP, sino que se han derivado con sonda uretral o cistostomía para paliar los síntomas obstructivos del tracto bajo. El IPSS postoperatorio fue considerablemente mejor comparado con el pre-quirúrgico con una media de 16,5 y solo 4 pacientes permanecieron con un IPSS severo a pesar de la cirugía, ninguno requirió sonda posterior a la intervención.

\section{Discusión}

El cáncer de próstata es el cáncer más frecuentemente diagnosticado en hombres después del cáncer de piel y es 
Tabla 1 Caracteristicas demograficas, oncologicas y resultados postoperatorios

\begin{tabular}{|c|c|}
\hline Variable & $N=19$ \\
\hline Media de edad (Años) & 73 \\
\hline Media hospitalización (Días) & 2,1 \\
\hline Antecedente pRTUP y nueva intervención & $5(26 \%)$ \\
\hline IPSS Inicial (Media) & $23,2 \pm 5,4$ \\
\hline IPSS severo & $17(89 \%)$ \\
\hline IPSS moderado & $2(11 \%)$ \\
\hline QoL (Media) & $4,1 \pm 1,4$ \\
\hline QMax inicial & $8,3 \pm 1$ \\
\hline \multicolumn{2}{|l|}{ TNM Inicial } \\
\hline T1C & $6(31 \%)$ \\
\hline $\mathrm{T} 2 \mathrm{~B}$ & $2(11 \%)$ \\
\hline$>\mathrm{T} 3$ & $6(31 \%)$ \\
\hline Desconocido & $5(26 \%)$ \\
\hline \multicolumn{2}{|l|}{ Enfermedad metastásica } \\
\hline Sí & $9(47 \%)$ \\
\hline No & $10(53 \%)$ \\
\hline Gleason Score inicial (Media) & 7,4 \\
\hline Score 6 & $5(26,3 \%)$ \\
\hline Score 7 & $3(15,7 \%)$ \\
\hline Score 8 & $4(21 \%)$ \\
\hline Score 9 & $7(36,8 \%)$ \\
\hline \multicolumn{2}{|l|}{ Tratamiento inicial } \\
\hline \multicolumn{2}{|l|}{ Radioterapia } \\
\hline Con intención curativa & $5(26,3 \%)$ \\
\hline Sin intención curativa & $14(73,6 \%)$ \\
\hline \multicolumn{2}{|l|}{ Terapia de privación androgénica } \\
\hline Sí & $14(73,6 \%)$ \\
\hline No & $5(26,3 \%)$ \\
\hline \multicolumn{2}{|l|}{ WHOQOL-BREF (Media) } \\
\hline Calidad de vida & 58,3 \\
\hline Salud física & 59,4 \\
\hline Salud psicológica & 57,5 \\
\hline Relaciones sociales & 67,1 \\
\hline Entorno & 62,2 \\
\hline IPSS Postoperatorio (Media) & 16,5 \\
\hline
\end{tabular}

la tercera causa de muerte por cáncer. El tratamiento para pacientes que no tienen opción curativa es la terapia de privación androgénica, bien sea por castración quirúrgica o farmacológica con el objetivo de aumentar la supervivencia y permitirles vivir con su enfermedad. En los hombres con carcinoma prostático los síntomas urinarios obstructivos son frecuentes, hasta el $82 \%$ pueden quejarse de ellos y afectar considerablemente su calidad de vida. Se pueden manejar mediante derivación de la vía urinaria o con tratamiento quirúrgico des-obstructivo mediante pRTUP, laser de diodo y
HPS 120 W Green laser. El objetivo de la cirugía es mejorar los síntomas obstructivos y evitar la necesidad de derivación permanente de la vía urinaria por los riesgos de infección del tracto urinario, calcificación y en teoría carcinoma no transicional de la vejiga., ${ }^{1,2}$

Gnanapragasam y col., llevaron a cabo en el 2006 un estudio retrospectivo en el que evaluaron a 46 hombres con cáncer de próstata TNM ( $\geq \mathrm{T} 2$ ), que estaban en terapia de privación androgénica y fueron llevados a pRTUP des-obstructiva por obstrucción del tracto de salida de la vejiga secundario al carcinoma prostático, comparando esa cohorte con 47 hombres sin evidencia histopatológica de cáncer de próstata que fueron llevados RTUP por síntomas urinarios obstructivos por primera vez.; evaluaron el IPSS, incontinencia urinaria, la necesidad de sonda y la necesidad de re-intervención un año después de la cirugía. Encontraron que el retiro de la sonda después de la cirugía fallo más frecuentemente en el grupo de pacientes con cáncer (43\% vs $10 \%$ ). El 37\% de los pacientes con cáncer de próstata requirieron una nueva intervención, necesitaron sonda por un periodo prolongado después de la cirugía y tuvieron tasas más altas de incontinencia urinaria. Encontraron que los pacientes que presentaron retención urinaria aguda (RUA), tuvieron mejores resultados, el $88 \%$ reportaron buenos resultados después de la pRTUP, un predictor de desenlaces desfavorables fue el cáncer de próstata resistente a la castración (CPRC) solamente un tercio de los pacientes tuvo un resultado favorable con la cirugía. Concluyen que dentro de la consejería a los hombres con cáncer de próstata se les debe incluir la advertencia tratamiento con pRTUP que no tiene tan buenos resultados como la RTUP para hiperplasia prostática benigna (HPB), y el riesgo de requerir derivación urinaria con sonda por un periodo prolongado es alto. En esa cohorte de pacientes, obtuvieron resultados comparables con los de nuestro estudio sin embargo, el fallo de retiro de sonda fue mayor que en el nuestro, y eso se puede deber a la volumetría prostática de los pacientes en esa serie, la cual no está reportada. 4

En un estudio similar llevado a cabo por Crain y col., donde evaluaron a 19 hombres con cáncer de próstata localmente avanzado llevados a 24 pRTUP con un tiempo medio desde el diagnostico hasta la pRTUP de $49 \pm 54$ meses, todos se encontraban con terapia de privación hormonal con agonistas de GNRH u orquidectomía y el volumen medio determinado por tacto rectal era de 38 gramos. A diferencia del estudio reportado anteriormente, 17 de los pacientes llevados a pRTUP en esa cohorte, había recibido radioterapia previamente y 2 tenían cáncer de próstata metastásico. Concluyeron que la pRTUP mejoró significativamente los síntomas urinarios obstructivos: el IPSS medio mejoro de 21 puntos a 11 después de la cirugía, aunque se requirieron más re-intervenciones en el grupo de pRTUP que en el grupo de RTUP por enfermedad benigna, 4 pacientes llevados a pRTUP requirieron tratamiento definitivo con derivación suprapúbica de la vía urinaria y se re-intervinieron en siete ocasiones cinco pacientes por persistir con síntomas urinarios obstructivos. Cabe resaltar que en este estudio, no se diferenció entre las técnicas de RTUP(Nesbit) y tunelización prostática (Orandi). Al final concluyen que la pRTUP en pacientes con cáncer de 
próstata localmente avanzado y obstrucción al tracto de salida de la vejiga es un procedimiento seguro, con baja morbilidad perioperatoria, mínimas perdidas sanguíneas y estancia hospitalaria corta, y en la que la mayoría de los pacientes mejoraron sus síntomas urinarios obstructivos con un impacto favorable en su calidad de vida. ${ }^{2}$

Se ha evaluado el tratamiento paliativo con láser de diodo por Javanmard y col. y HPS 120 W Green laser por Chen y col. En la cohorte del láser de diodo, se aleatorizaron a los pacientes con cáncer de próstata avanzado y síntomas urinarios obstructivos en dos grupos, uno que recibió tratamiento desobstructivo con pRTUP y el otro con láser de diodo. Siguieron los pacientes hasta 6 meses después de la cirugía con IPSS, residuo postmiccional (RPM) y Qmax. Encontraron que hubo una mejoría significativa en IPSS, disminución del RPM y mejoría en el flujo libre; no hubo diferencia estadísticamente significativa entre las dos intervenciones quirúrgicas de las variables descritas pero si lo hubo en cuanto a la estancia hospitalaria y el uso de catéter fue menor en el grupo tratado con láser de diodo. En cuanto al estudio de pacientes con cáncer de próstata localmente avanzado con RUA por medio de HPS 120 W Green laser, evaluaron 39 pacientes a los 6 y 12 meses. Encontraron mejoría significativa en IPSS, Qmax, RPM en todos los controles de seguimiento, el tiempo medio de cateterismo fue 3,3 días y el tiempo medio de hospitalización fue 5,2 días, por lo que concluyeron que el tratamiento paliativo de pacientes con cáncer de próstata localmente avanzado con HPS $120 \mathrm{~W}$ Green laser es seguro y efectivo.,

Además del tratamiento de los síntomas urinarios obstructivos en el paciente con cáncer de próstata localmente avanzado, se ha propuesto la citoreducción tumoral paliativa para mejorar la respuesta a la terapia de privación androgénica en el cáncer de próstata metastásico hormonosensible. Qin y col., evaluaron 39 pacientes con cáncer de próstata que venían en tratamiento con bloqueo androgénico completo y que fueron llevados a pRTUP como tratamiento de los síntomas urinarios obstructivos, y los compararon con 107 pacientes con cáncer de próstata metastásico hormonosensible que venían en tratamiento con bloqueo androgénico completo y que no requirieron intervención quirúrgica. Encontraron que: el nadir del PSA fue menor en el grupo manejado con pRTUP, un número mayor de pacientes en el grupo de solo bloqueo androgénico desarrollo refractariedad a la terapia de privación hormonal y que el grupo de pRTUP tuvo mayor supervivencia global y mayor supervivencia enfermedad específica, aunque ese punto no fue estadísticamente significativo. ${ }^{7}$

Al evaluar los resultados de nuestro estudio y compararlos con los estudios mencionados anteriormente, podemos concluir que son comparables y que apoyan la premisa de que la RTUP paliativa es la mejor alternativa en pacientes con cáncer de próstata localmente avanzado o metastasico y uropatía obstructiva secundaria a crecimiento prostático, sin embargo, debemos resaltar que las limitaciones de nuestro estudio no son desconocidas por los investigadores $\mathrm{y}$ radican en que se trata de un estudio con una cohorte pequeña de pacientes, es un estudio observacional descriptivo, retrospectivo que no permite realizar más que un análisis univariado de las variables; sin embargo el propósito de este estudio radica en presentar una serie de casos exitosos de pRTUP en el manejo de esos pacientes. Consideramos que se deben realizar futuros estudios aleatorizados que evalúen la pRTUP, el green laser, el láser de diodo y el manejo conservador para definir cuál es el mejor abordaje en ese grupo de pacientes.

\section{Conclusiones}

La pRTUP se afianza como una intervención válida para la uropatía obstructiva secundaria a cáncer de próstata. Nos permite confirmar en nuestra población los resultados obtenidos en otros estudios con series más grandes. Se observa que aunque los cambios objetivos en el IPSS son muy significativos, la calidad de vida en los parámetros de salud física y psicológica no varían significativamente, debiéndose probablemente a la noción de enfermedad en los pacientes oncológicos o al hecho de tener que vivir con distintas comorbilidades o factores ambientales y sociales que de por sí, afectan la calidad de vida. En quienes se derivan con sonda uretral o suprapúbica, que es la alternativa de tratamiento más frecuentemente utilizada, no se persigue recuperar la dinámica miccional, al tiempo que la calidad de vida se presume más comprometida, surge proponer esa opción de tratamiento como una alternativa a la derivación permanente de la vía urinaria, que de acuerdo con los resultados obtenidos es segura, factible y mejora considerablemente la dinámica miccional y la calidad de vida. Queda por resolver si pudiera ser una alternativa para establecerse en casos de obstrucción severa que aún no se encuentran derivados con sonda uretral.

Conflicto de interés

Declaramos que ninguno de los autores presenta conflicto de interés.

Protección de personas y animales

Los autores declaran que para esta investigación no se han realizado experimentos en seres humanos ni en animales.

\section{Confidencialidad de los datos}

Los autores declaran que han seguido los protocolos de su centro de trabajo sobre la publicación de datos de pacientes.

Derecho a la privacidad y consentimiento informado Los autores han obtenido el consentimiento informado de los pacientes y/o sujetos referidos en el artículo. Este documento obra en poder del autor de correspondencia.

\section{Bibliografía}

1 Marszalek M, Ponholzer A, Rauchenwald M, Madersbacher S. Palliative transurethral resection of the prostate: functional outcome and impact on survival. BJU Int 2007;99(01):56-59

2 Crain DS, Amling CL, Kane CJ. Palliative transurethral prostate resection for bladder outlet obstruction in patients with locally advanced prostate cancer. J Urol 2004;171(2 Pt 1):668-671 
3 Gnanapragasam VJ, Kumar V, Langton D, Pickard RS, Leung HY. Outcome of transurethral prostatectomy for the palliative management of lower urinary tract symptoms in men with prostate cancer. Int J Urol 2006;13(06):711-715

4 Thomas DJ, Balaji VJ, Coptcoat MJ, Abercrombie GF. Acute urinary retention secondary to carcinoma of the prostate. Is initial channel TURP beneficial? J R Soc Med 1992;85(06):318-319

5 Chen D, Xue B, Shan Y, Yang D, Sun C, Gao J. GreenLight HPS 120-W laser photoselective vaporization of the prostate as early therapy for acute urinary retention in advanced prostate cancer patients. Lasers Med Sci 2013;28(05):1339-1344
6 Javanmard B, Hassanzadeh Haddad A, Yaghoobi M, Lotfi B. Diode laser ablation of prostate and channel transurethral resection of prostate in patients with prostate cancer and bladder outlet obstruction symptoms. Urol J 2014;11(04): $1788-1792$

7 Qin XJ, Ma CG, Ye DW, et al. (2012, April). Tumor cytoreduction results in better response to androgen ablation-a preliminary report of palliative transurethral resection of the prostate in metastatic hormone sensitive prostate cancer. In Urologic Oncology: Seminars and Original Investigations (Vol. 30, No. 2, pp. 145-149). Elsevier. 